\title{
MEHMO (Mental retardation, Epileptic seizures, Hypogenitalism, Microcephaly, Obesity): a new $\mathrm{X}$-linked mitochondrial disorder
}

\author{
Esther Leshinsky-Silver ${ }^{1,2}$, Ami Zinger ${ }^{3}$, Chaim N Bibi $^{4}$, Varda Barash ${ }^{5}$, Menachem Sadeh $^{6}$, \\ Dorit Lev $^{2}$ and Tally Lerman Sagie ${ }^{*, 2}$
}

${ }^{1}$ Molecular Genetics laboratory and Institute of Physiologic Hygiene, Wolfson Medical Center, Holon, Israel; ${ }^{2}$ The Mitochondrial Disease Clinic, Metabolic Neurogenetic Unit, Wolfson Medical Center, Holon, Israel; ${ }^{3}$ Genetic Institute, Kaplan Medical Center, Rechovot, Israel; ${ }^{4}$ Pediatric Intensive care Unit, Barzilai Medical Center, Ashkelon, Israel; ${ }^{5}$ Biochemistry Department, Hadassa Medical Center, Jerusalem, Israel; ${ }^{6}$ Department of Neurology, Wolfson Medical Center, Holon, Israel

MEHMO (Mental retardation, Epileptic seizures, Hypogenitalism, Microcephaly and Obesity) is an X-linked disorder characterised by mental retardation, epileptic seizures, hypogenitalism, microcephaly and obesity. It was recently assigned to the locus Xp21.1-p22.13. We describe a child with MEHMO and lactic acidosis whose muscle biopsy revealed markedly reduced activities of complexes 1,3 and 4 of the mitochondrial electron transport chain. Histological staining showed mitochondrial proliferation and lipid storage. Electron microscopy revealed abnormal and enlarged mitochondria with concentric cristae and electron dense bodies. This is the first identification of MEHMO as a mitochondrial disorder and one of the very few X-linked mitochondrial syndromes.

European Journal of Human Genetics (2002) 10, 226 - 230. DOI: 10.1038/sj/ejhg/5200791

Keywords: MEHMO; mtDNA; X-linked mental retardation; respiratory chain

\section{Introduction}

MEHMO (Mental retardation, Epileptic seizures, Hypogenitalism, Microcephaly, Obesity) was first described as a new syndrome by Delozier-Blanchet et al. ${ }^{1}$ but was named by Steinmuller et al. ${ }^{2}$ It is characterised by profound mental retardation, microcephaly, hypogonadism, epilepsy and obesity. Haplotype and linkage analysis in a large threegeneration family recently assigned the disease locus to Xp21.1-Xp22.13 in a region flanked by CYBB and DXS365. ${ }^{2,3}$ The multisystem involvement in this syndrome is typical of mitochondrial diseases where the defect in energy production may damage all organs. Although hypogonadism and obesity are not usually described in mitochondrial diseases,

${ }^{*}$ Correspondence: T Lerman Sagie, Metabolic Neurogenetic Unit, Wolfson Medical Center, Holon, Israel, Tel: 0119723502 8458; Fax: 0119723 5028566; E-mail: asagie@post.tau.ac.il

Received 20 November 2001; revised 23 January 2002; accepted 31 January 2002 microcephaly, epileptic seizures, mental retardation are very characteristic.

We investigated the mitochondrial involvement in a child with MEHMO and lactic acidaemia. Mitochondrial morphology (both by light and electron microscopy) and biochemical function (respiratory enzyme activity) confirmed that MEHMO is a mitochondrial disorder.

\section{Material and methods}

Case report

The patient was the product of a normal 40 weeks pregnancy and delivery to healthy, non-consanguineous Ethiopian Jews. His weight was $2.710 \mathrm{Kg}$ and head circumference was $31.5 \mathrm{~cm}$.

Microcephaly was first noted at the age of 2 months when he was hospitalised for pneumonia. A head CT was normal. At the age of 11 months he was hospitalised for infantile spasms. The EEG revealed a burst suppression pattern. He was treated with vigabatrin and ACTH. Metabolic evaluation 
revealed elevated blood lactate and urinary 3-methylglutaconic acid. He was referred for a muscle biopsy and insertion of a gastrostomy tube at the age of 12 months. Examination was significant for an obese infant with extreme microcephaly (head circumference $40 \mathrm{~cm}$ ), an amphore- like facies, sloping forehead, long eyelashes, large lips, no teeth and micropenis (Figure 1).

Neurologic examination revealed a hypotonic baby who did not fix or follow, despite a normal eye exam. There were no facial movements and constant drooling. He had neither head control nor any spontaneous movements. No reflexes were elicited. He reacted to pain only by moving his fingers.

Family history The parents have three healthy sons. The mother's mother had two sons who died in their first year of life.

\section{Methods}

Biochemical studies Five per cent muscle homogenate in $0.25 \mathrm{~m}$ sucrose, $20 \mathrm{~mm}$ Tris, $\mathrm{pH}$ 7.2, $40 \mathrm{~mm} \mathrm{KCl} 2 \mathrm{~mm}$ EGTA and $1 \mathrm{mg} / \mathrm{ml}$ albumin was prepared in Teflon-glass homo-

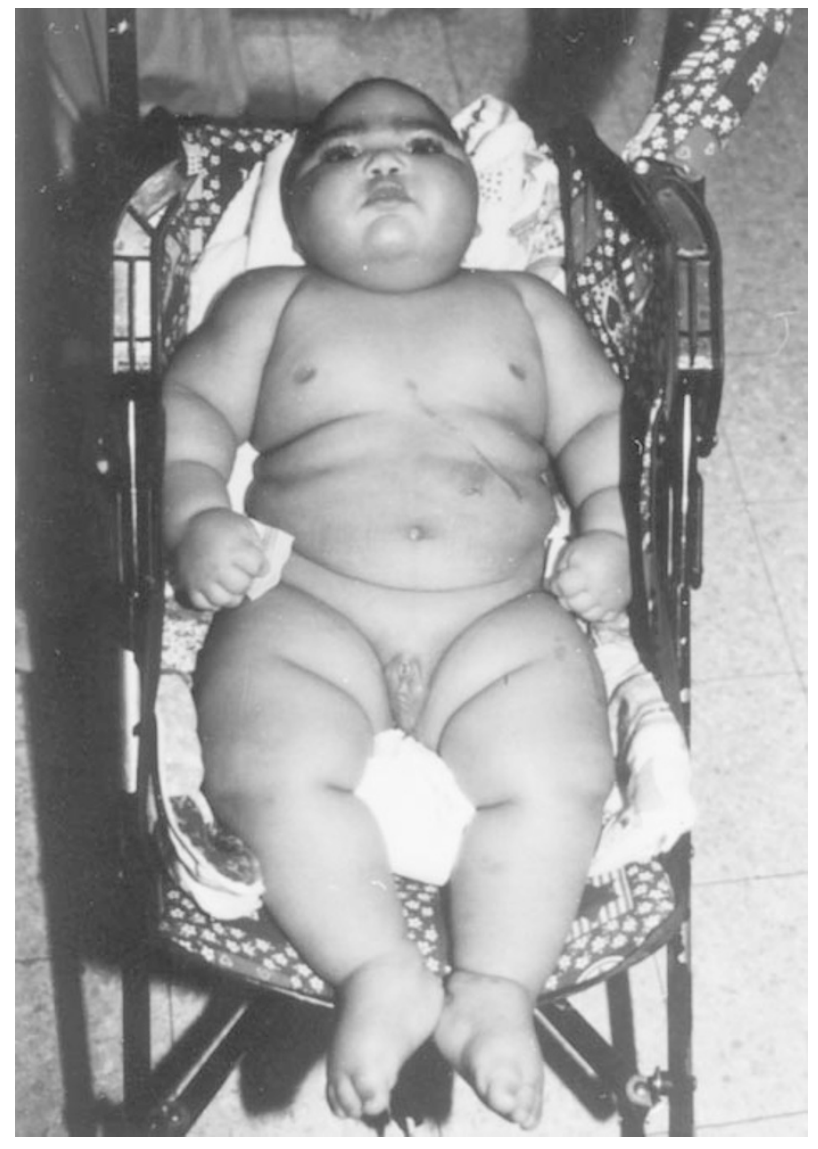

Figure 1 Patient at the age of one year. Note hypogenitalia, obesity and microcephaly. genizer from frozen specimens. The homogenate was centrifuged at $600 \times g$ for $10 \mathrm{~min}$ and the supernatant was used for enzyme determinations after being frozen/thawed (three times) in liquid nitrogen.

Respiratory chain enzyme activities of complexes I+III (rotenone-sensitive $\mathrm{NADH}$ cytochrome c oxidoreductase), II+III (antimycine-sensitive succinate -cytochrome c oxidoreductase), II (succinate dehydrogenase), IV (cytochrome c oxidase) and activity of the marker enzyme citrate synthase in skeletal muscle were measured spectrophotometrically as described previously. ${ }^{4}$

\section{Histological and electron microscopy analysis}

Ten micrometer of the muscle sections were stained with haematoxylin and eosin, modified Gomori trichrome, PAS, Sudan Black B, NADH-tetrazolium reductase, succinate dehydrogenase, cytochrome oxidase, ATPase at pH 9.4 and after preincubation at $\mathrm{pH} 4.3$ and 4.6. An additional specimen was fixed in $4 \%$ glutaraldehyde in phosphate buffer and prepared for electron microscopy by standard methods.

\section{Results}

OXPHOS activity

Complex I+III, II+III, and IV were markedly reduced while complex II, citrate synthase, PDH and lipoamide dehydrogenase (E3) were within the normal values (Table 1). The ratio of I+III/CS, II+III/CS and IV/CS are more significantly reduced. Thus, there are only deficiencies in complexes that are partially encoded by mtDNA. Complex II, the only complex exclusively encoded by nuclear DNA was not affected.

\section{Skeletal muscle histology and ultrastructure}

The most prominent finding was the presence of abundant fatty infiltration surrounding muscle fascicles (Figure 2a). The fascicles were of different sizes, and some fat was also

Table 1 Respiratory chain enzymes and pyruvate dehydrogenase complex activities in muscle homogenates of the patient and normal controls

\begin{tabular}{lccc}
\hline & & \multicolumn{2}{c}{ Normal controls } \\
Enzyme & Patient & Range & Mean \pm SD \\
\hline Complex I+III & 6.1 & $10-40$ & $18 \pm 5.9$ \\
Complex II+III & 10 & $12-30$ & $17 \pm 4$ \\
Complex II & 64 & $30-60$ & $44 \pm 10$ \\
Complex IV & 38 & $60-160$ & $107 \pm 41$ \\
Citrate synthase (CS) & 266 & $70-350$ & $120 \pm 30$ \\
PDH complex & 3.2 & $1.9-4.5$ & \\
Lipoamide dehydrogenase (E3) & 222 & $45-150$ & \\
I+III/CS & 0.02 & & $0.15 \pm 0.05$ \\
II+III/CS & 0.03 & & $0.14 \pm 0.05$ \\
II/CS & 0.24 & & $0.36 \pm 0.14$ \\
IV/CS & 0.14 & & $0.89 \pm 0.22$ \\
\hline ACtivtes & axpressed
\end{tabular}

Activites are expressed in $\mathrm{nmol} / \mathrm{min} / \mathrm{mg}$ protein. 
a

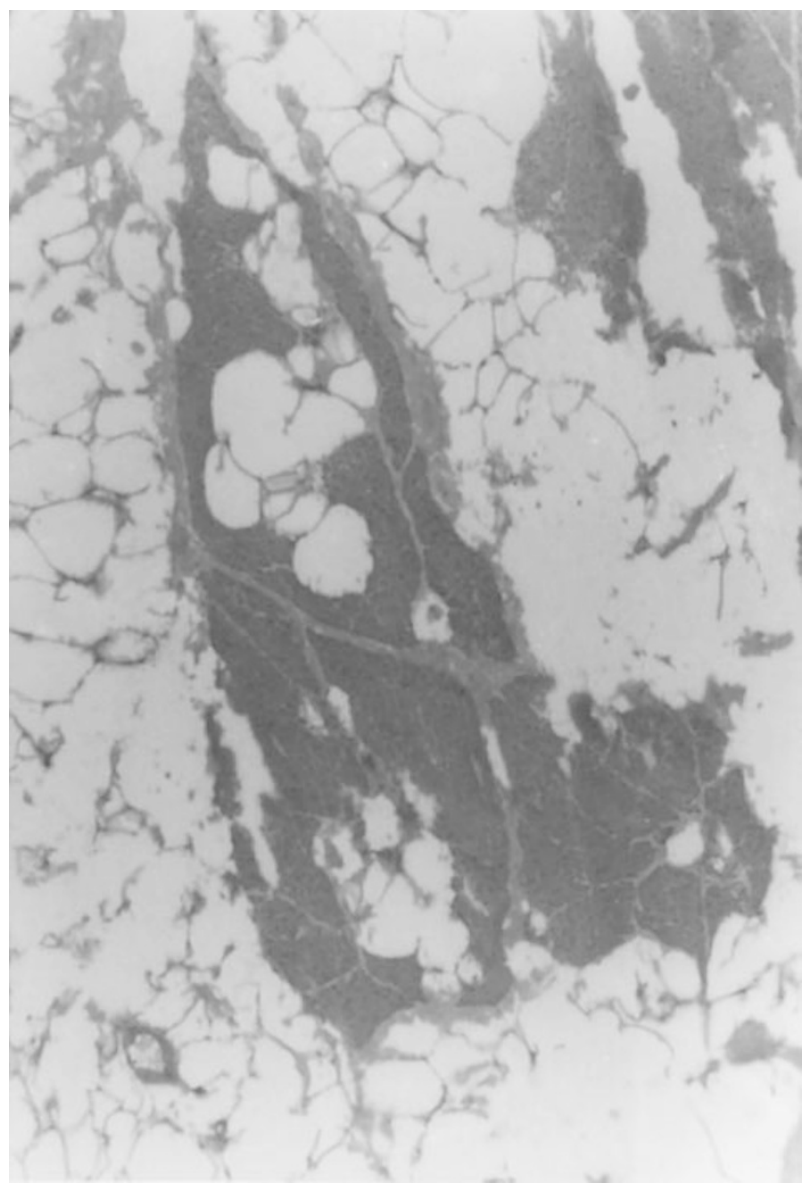

b

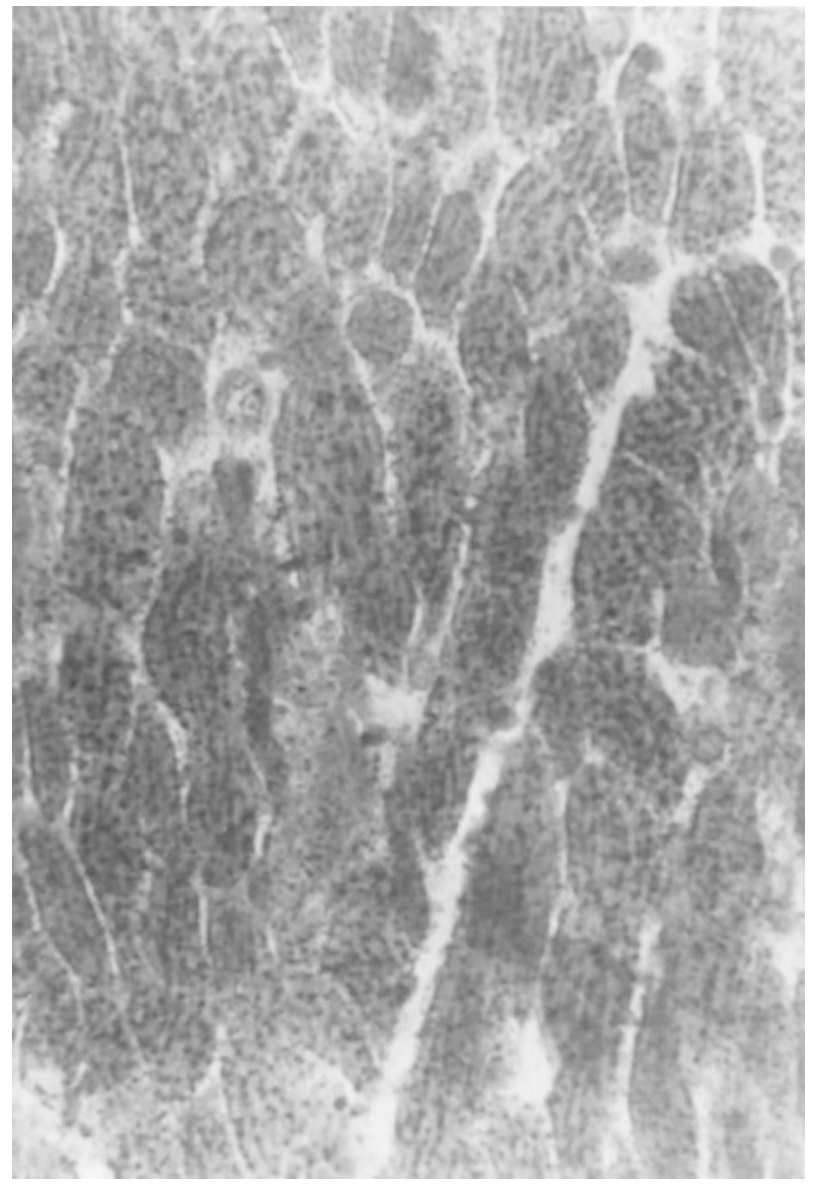

Figure 2 (a) H\&E stain. A muscle fascicle contains several areas of fat, surrounded by massive fatty infiltration. (b) Sudan Black B stain. Muscle fibres contain numerous lipid droplets.

seen in between muscle fibres. Sudan Black B stain showed large lipid droplets within muscle fibres (Figure $2 \mathrm{~b}$ ).

COX, SDH and NADH staining showed excessive mitochondria that were distributed through the whole area of the fibres, and not just in the periphery. Fibre-type distribution was normal. No ragged-red fibres were seen with the trichrome stain. Electron microscopy analysis (Figure 3) showed either concentric arrangement of the cristaes in huge mitochondria or irregular lammelar arrangement (Figure 3a). Electron dense bodies were seen inside the mitochondria. Some of the mitochondria were huge (Figure 3b). There were no paracrystalline inclusions.

\section{Discussion}

The combination of clinical symptoms including epileptic seizures, hypogenitalism, microcephaly and obesity is identical to those described by Steinmuller et al. ${ }^{2}$ in patients having MEHMO syndrome. Steinmuller et al's patients also had a fatty liver and elevated fatty acids and acylcarnitine in blood. Our patient did not undergo a liver biopsy but his muscle biopsy revealed marked fat infiltration thus also suggesting an abnormality in fat metabolism. DeLozierBlancher et $a l^{1}$ described two patients who did not have seizures but were described as having highly abnormal EEG as well as abnormal neurological status. In all the previously described patients, a muscle biopsy was not performed and plasma lactate is not mentioned. In our patient, elevated plasma lactate level, associated with seizures and progressive microcephaly, a picture previously described in Alpers syndrome, ${ }^{6}$ lead us to obtain a muscle biopsy and to assay the respiratory chain enzymes.

The multiple deficiencies of mtDNA encoded respiratory chain complexes (I+III, II+III, and IV) with normal levels of nuclear encoded mitochondrial enzymes (citrate synthase, $\mathrm{PDH}, \mathrm{E3}$, and complex II) and the morphology of the mitochondria suggests a nuclear defect that leads to abnormal mitochondria function.

We were unable to do linkage analysis studies in our family to prove X-linked inheritance. The family history of two male 
a

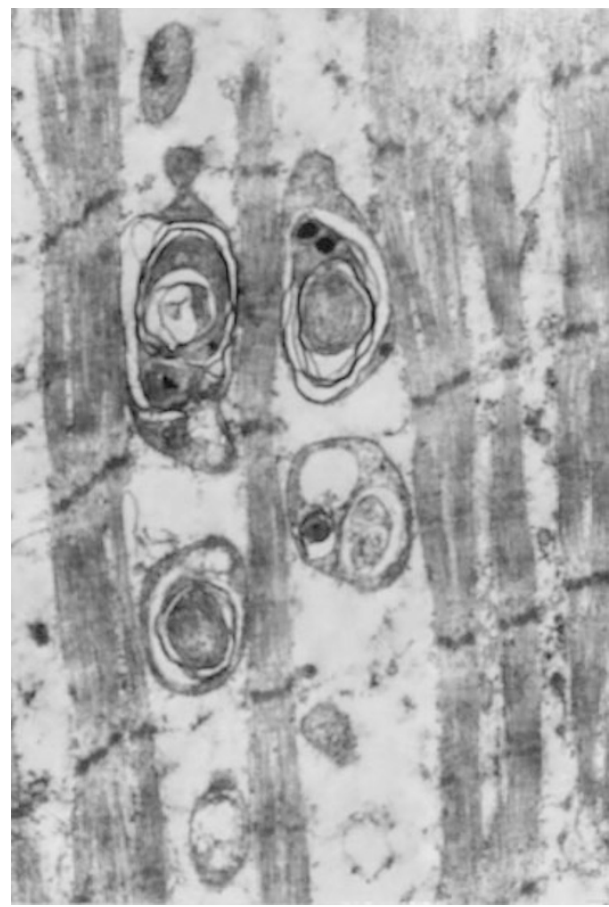

b

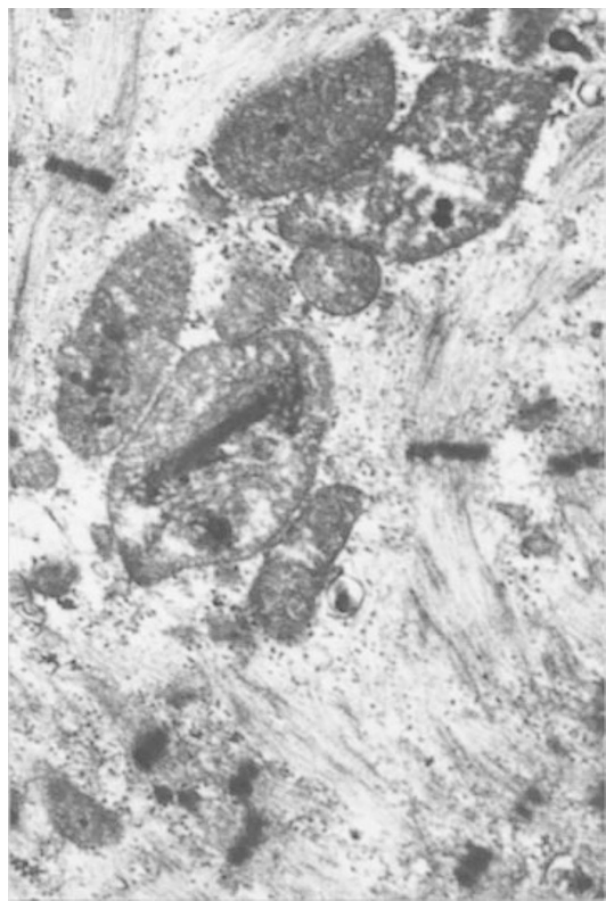

Figure 3 Electron microscopy of muscle biopsy. (a) Concentric arrangement of the cristae and irregular lammelar arrangement. (b) Electron dense bodies are seen in huge mitochondria.

sibling of the mother who died in the first year of life (although not proven to be affected by the same disease) and the similarity of our patient to those described previously ${ }^{2}$ suggests that our patient also has the X-linked MEHMO syndrome. If the maternal uncles died of another disease and having three unaffected boys in the same generation, then we are dealing with a sporadic form of respiratory chain.

There are only a few X-linked mitochondrial disorders. Mohr-Tranebjaerg (Sensorineural deafness, blindness, and movement disorder) is caused by mutations in the DDP1 gene, which is involved in the mitochondrial transport machinery. ${ }^{5}$ Sideroblastic anemia with ataxia is another $\mathrm{X}$ linked disorder, which is caused by mutations in the ABC-7 gene, which is responsible for the assembly of FeS clusters from the mitochondria into cytoplasmic proteins. ${ }^{6}$

Barth syndrome is the third X-linked mitochondrial disorder characterised by dilated cardiomyopathy. This syndrome is caused by mutations in the G4.5 gene (tafazzin) whose function is still unknown. ${ }^{7}$

Complex I is composed of 43 subunits, one of which (NDUFA1) is encoded on the X chromosome (Xq24-25). ${ }^{8}$ Thus far no mutations have been reported in this subunit in isolated complex I deficiency. Mutations in the NDUFA1 is not relevant in our patient since he had multiple respiratory chain deficiencies and the disorder is linked to different locus on the X-chromosome.
The unique clinical presentation of obesity and fat infiltration in the liver can be due to a secondary inhibition of fat catabolism due to a decrease in activity of respiratory chain enzymes or could derive from a microdeletion which involves mitochondrial function and fat metabolism genes.

This finding of mitochondrial dysfunction in MEHMO needs to be confirmed in other patients. We are now focusing our attention on finding a candidate mitochondrial gene in the $\mathrm{Xp} 21.1-\mathrm{Xp} 21.13$ region that might be involved in this unique syndrome.

\section{References}

1 Delozier-Blanchet CD, Haenggeli CA, Engel E: Microcephalic nanism, severe retardation, hypertonia, obesity, and hypogonadism in two brothers: a new syndrome? J Genet Hum 1989; 37: $353-365$.

2 Steinmuller R, Steinberger D, Muller U: MEHMO (mental retardation, epileptic seizures, hypogonadism and genitalism, microcephaly, obesity), a novel syndrome: assignment of disease locus to Xp21.1-p22.13. Eur J Hum Genet 1998; 6: 201 206.

3 Delozier-Blanchet CD, Haenggeli CA, Bottani A: MEHMO, a novel syndrome: assignment of disease locus to Xp21.1-p22.13. A letter. Eur J Hum Genet 1999; 7: 621-622.

4 Rustin P, Chretien D, Bourgeron $\mathrm{T}$ et al: Biochemical and molecular investigations in respiratory chain deficiencies. Clin Chim Acta 1994; 228: 35 -51. 
5 Jin H, May M, Tranebjaerg L et al: A novel X-linked gene, DDP, shows mutations in families with deafness (DFN-1), dystonia, mental deficiency and blindness. Nat Genet 1996; 14; 177 180.

6 Bekri S, Kispal G, Lange $\mathrm{H}$ et al: Human ABC7 transporter:gene structure and mutations causing X-linked sideroblastic anemia with ataxia with disruption of cytosolic iron-sulfur protein maturation. Blood 2000; 96: 3256-3263.
7 Bione S, D'Adamo P, Maestrini E et al: A novel X-linked gene, G4.5. is responsible for Barth syndrome. Nat Genet 1996; 12: $385-389$.

8 Zhuchenko O, Wehnert M, Bailey J et al: Isolation, mapping and genomic structure of an X-linked gene for a subunit of the human mitochondrial complex I. Genomics 1996; 37: 281-288. 